\title{
Error Robust Scalable Audio Streaming Over Wireless IP Networks
}

\author{
Qian Zhang, Senior Member, IEEE, Guijin Wang, Zixiang Xiong, Senior Member, IEEE, Jianping Zhou, and \\ Wenwu Zhu, Senior Member, IEEE
}

\begin{abstract}
Streaming high-fidelity audio over wireless Internet protocol (IP) networks is a challenging task because the networks present not only packet losses, but also residual bit errors. These losses and errors have severe adverse effect on the compressed audio bitstream. To solve this problem, this paper introduces error resilience in conjunction with error protection for scalable audio streaming over wireless networks. Specifically, error resilience is achieved by performing bitstream data partitioning and reversible variable length coding in the audio coder. Error protection is provided by layered product channel code to simultaneously handle packet losses and residual bit errors. Both the row and column codes of the product code provide unequal error protection for different layers of the audio bitstream by considering the characteristics of the scalable audio. Rate-distortion optimization is performed to determine the best source-channel coding tradeoff that minimizes the average expected end-to-end distortion. Simulation results demonstrate the effectiveness of our proposed approach.
\end{abstract}

Index Terms-Bit allocation, error resilience, error robustness, rate-distortion optimization, scalable audio streaming, unequal error protection, wireless IP networks.

\section{INTRODUCTION}

W ITH the emerging third-generation (3G) wireless technology, streaming high-fidelity audio over wireless channels is becoming a reality. Meanwhile, Internet protocol (IP) based architecture for wireless systems promises to provide next-generation wireless services such as voice, high-speed data, Internet access, audio and video streaming on an all IP network [1]. Real-time audio streaming has bandwidth requirement and can only tolerate very few errors. Moreover, bandwidth fluctuates in wireless channels present new challenges to audio streaming. Thus, it is important that an audio streaming scheme has the ability to adapt the audio bit rate according to network conditions. With scalable audio compression [3], [4], a single compressed bitstream can be generated with different subsets of it corresponding to the compressed version of the same audio clip at various rates. This is very beneficial for audio delivery over time-varying networks with bandwidth fluctuation.

Manuscript received October 8, 2002; revised March 19, 2003. The associate editor coordinating the review of this manuscript and approving it for publication was Dr. Nelson L. S. Fonseca.

Q. Zhang, J. Zhou, and W. Zhu are with Microsoft Research Asia, Haidian District, Beijing 100080, China (e-mail: qianz@microsoft.com, jzhou2@uiuc.edu).

G. Wang is with the Department of Electronic Engineering, Tsinghua University, Beijing 100084, China (e-mail: wanggj98@mails.tsinghua.edu.cn).

Z. Xiong is with the Department of Electrical Engineering, Texas A\&M University, College Station, TX 77843 USA (e-mail:zx@lena.tamu.edu).

Digital Object Identifier 10.1109/TMM.2004.837249
Four quality-of-service classes are defined in the 3GPP specifications [2]. Two important metrics for each class are the average residual bit-error rate (BER) and average packet loss rate, where the former indicates the undetected BER in the delivered service data units (SDUs). For streaming class supported in 3GPP [31], the average residual BER varies from $5 * 10^{-2}$ to $10^{-6}$, and the average packet loss rate goes from $10^{-1}$ to $10^{-5}$. Thus, for audio streaming over 3G IP networks, the bitstream will experience not only packet loss, but also residual bit errors. Like in other wireless multimedia applications, efficient compression techniques must be employed to meet bandwidth limitations in audio streaming. Source compression is achieved at the cost of sensitivity to transmission errors, which have severe adverse effects on decompressing the received bitstream and sometimes completely crash the decoder.

Two techniques have been proposed in the literature to deal with transmission errors: error resilience and error protection [14]. The former is usually deployed at the source coding level. It aims to detect and locate errors, support resynchronization and prevent information losses in the transmission process. Lack of error resilience results in error propagation. With error resilience coding, good audio quality can be obtained when the BER is at the low end of the $\left[10^{-6}, 5 * 10^{-2}\right]$ range [13]. Thus acceptable audio quality is usually not obtainable with error-resilient coding alone and error protection techniques are necessary to ensure high-quality audio streaming over 3G IP networks.

In this paper, we present an end-to-end architecture for the above application and focus on designing error robustness tools for scalable audio streaming. We first introduce an error-resilient scalable audio coding (ERSAC) [13] technique that features data partitioning and reversible variable length codes (RVLC). The former is used to reduce error propagation among data segments; the latter is to locate errors and minimize error propagation. We then propose layered product channel codes to mitigate the effect of packet loss and bit errors for error protection. Both the row and column codes provide unequal error protection (UEP) for different layers of the scalable audio bitstream. The proposed error protection technique is very flexible in the sense that it handles different channel conditions. To find the optimal UEP, we build an error model for wireless IP networks to estimate the channel condition based on feedback information collected from different protocol layers. Under a given channel condition, we establish an operational rate-distortion function to find the optimal source-redundancy tradeoff that minimizes the expected end-to-end distortion.

The rest of this paper is organized as follows. Section II briefs the channel model of wireless IP network. Section III describes 
the architecture for scalable audio streaming over wireless networks. Section IV introduces ERSAC. Section V details the layered product code structure for error protection. Section VI establishes the operational rate-distortion function for our scalable audio streaming system, together with the optimal joint source-channel coding solution. Section VII covers simulation results and Section VIII concludes this paper.

\section{A. Previous Work}

Although many works have been done on error resilient video coding [6], there are few reports on error resilient audio coding in the literature. Error resilient schemes for video coding cannot be directly ported to audio coding because the characteristics of audio and video are different. There exists strong correlation among adjacent video frames that can be exploited in error concealment. In contrast, there is almost no correlation between adjacent audio frames, which typically are about $400 \mathrm{~ms}$ long. Moreover, audio coding artifacts caused by corrupted frames are very annoying to human ears.

Several error protection schemes had been studied for audio streaming over the Internet and wireless channels. Error control schemes based on forward error correction (FEC) are proposed in [9], [10] for audio streaming over the Internet. Yung et al. described a fixed UEP scheme for MPEG audio over wireless channels [7], [8]. However, neither of these schemes considers time-varying channel conditions or handles both packet losses and residual bit errors. There are, however, several error control schemes proposed for image transmission over channels with both packet losses and bit errors [11], [12]. Sachs et al. used multiple-description product codes for transmission of SPIHT coded images [11]. A rate-compatible punctured convolutional (RCPC)/cyclic redundancy check (CRC) concatenated channel coder is proposed for embedded image transmission over channels with both bit errors and packet erasures [12]. Neither of the schemes in [11], [12] discusses the interaction between the column and row channel codes or how to allocate the varying network bandwidth between source coding and channel coding. Moreover, these error control schemes assume that RCPC codes can correct all errors, which is not true in real wireless network scenarios.

\section{Channel Characteristics And Models FOR WIRELESS IP NETWORKS}

In wireless IP networks, according to specifications of packet data convergence protocol (PDCP), radio link control (RLC), and medium access control (MAC) layers [28], received SDUs with bit errors are discarded, resulting in packet loss at the IP layer. In addition, data in packets received by the receiver may also experience residual bit errors that cannot be detected by CRCs. In the following, we describe the channel model that we use to characterize both packet lose and residual bit errors.

\section{A. Packet Loss Model}

Zorzi et al. investigated the accuracy of a first-order Markov process in modeling transmission on a correlated Rayleigh fading channel in the data link layer [16]. In a companion work [17], we approximate the success and/or failure of a sequence of transmission packets by a two-state Markov chain, whose transition matrix is given by $A=\left[\begin{array}{cc}p & 1-q \\ 1-p & q\end{array}\right]$, where $p$ (or $q$ ) is the probability that the $j$ th packet transmission is successful, conditioning on the $(j-1)$ th packet transmission being successful (or unsuccessful). The transition matrix $A$ completely characterizes the channel properties. The steady state probability $P_{L}$ that a packet loss occurs is $P_{L}=(1-p) /(2-p-q)$. Maximum likelihood (ML) estimators for $p$ and $q$ can be formed as

$$
\hat{p}=\frac{N_{1}}{\left(N_{1}+N_{2}\right)}, \quad \hat{q}=\frac{N_{3}}{\left(N_{3}+N_{4}\right)}
$$

where $N_{1}\left(N_{2}\right)$ is the number of successfully received (lost) packets when the previous packet is lost and $N_{3}\left(N_{4}\right)$ is the number of lost (successfully received) packets when the previous packet is successfully received, respectively. These parameters can be measured in the application layer at the receiver side of our proposed end-to-end streaming system (see Section III).

\section{B. Bit-Error Model}

The radio channel discussed in this work is characterized by Rayleigh fading, which can be also modeled by a Markov process [18]. As mentioned earlier, after an IP packet passes through the physical layer and data link layer, residual bit errors may exist. Although techniques such as channel coding and interleaving can be used to combat noise in the air, the remaining residual errors are still bursty in nature. We thus model them with a two-state Markov chain.

Under this channel model, the physical channel assumes one of two states: a "good" state $G$ and a "bad" state $B$. State $G$ represents the channel in a "good" state, characterized by a very low BER $\operatorname{Pe}(G)$. State $B$ indicates that the channel is operating under fading conditions with higher BER $P e(B)$. The transition probability from state $G$ to $B$ is denoted by $P_{G B}$ and the transition probability from state $B$ to $G$ is denoted by $P_{B G}$. Then important channel properties such as BER and fading depth, can be characterized by the transition matrix $P=\left(\begin{array}{cc}1-p_{G B} & P_{G B} \\ P_{B G} & 1-P_{B G}\end{array}\right)$. Based on this model, the steady-state probability that the channel is in state $B$ is given by $\varepsilon=P_{G B} /\left(P_{B G}+P_{G B}\right)$ and the average BER by $\mathrm{BER}=\varepsilon P_{e}(B)+(1-\varepsilon) P_{e}(G)$.

\section{AN END-TO-END ARCHITECTURE FOR SCALABLE AUdIO STREAMING}

Fig. 1 depicts the block diagram of our proposed end-to-end architecture for scalable audio streaming over wireless IP networks. Key components in our proposed architecture include ERSAC, channel-adaptive layered product codes for error protection, and optimal bit allocation for error robustness. This section gives an overview of the system; Sections IV-VI detail each of these components.

In our proposed system, the raw audio signal is first compressed into a layered bitstream using a scalable audio coder at the sender side. ERSAC is the first component used to combat transmission errors in the system. It consists of data 


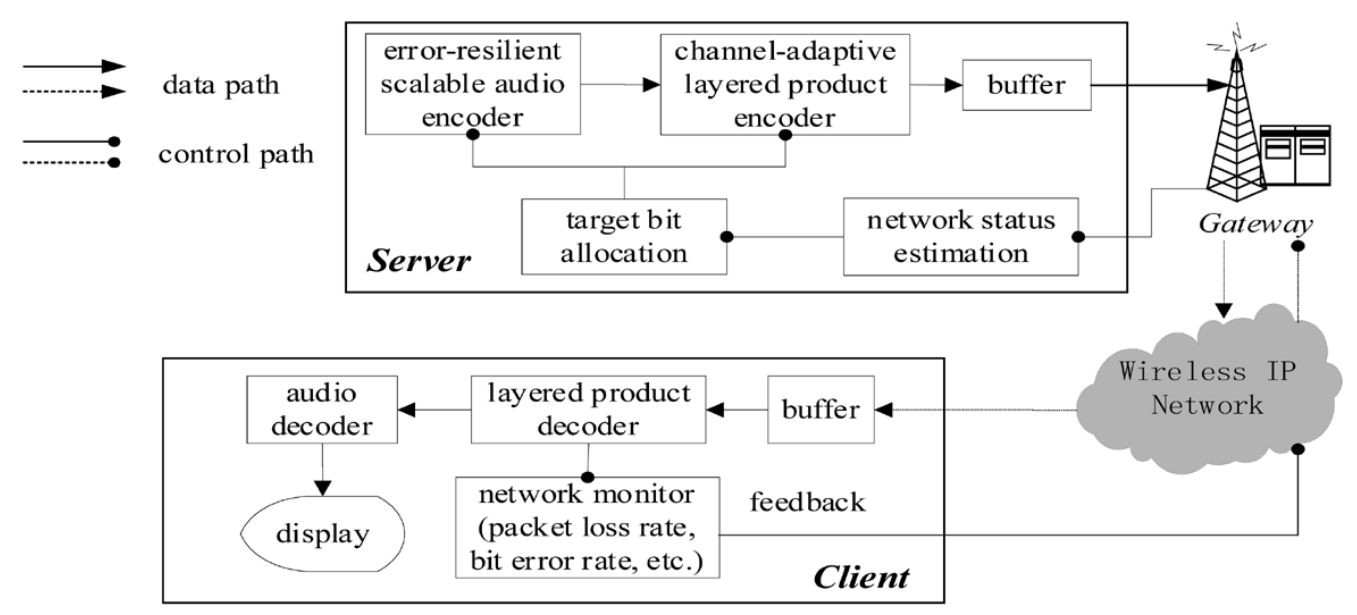

Fig. 1. End-to-end architecture for scalable audio streaming over wireless networks.

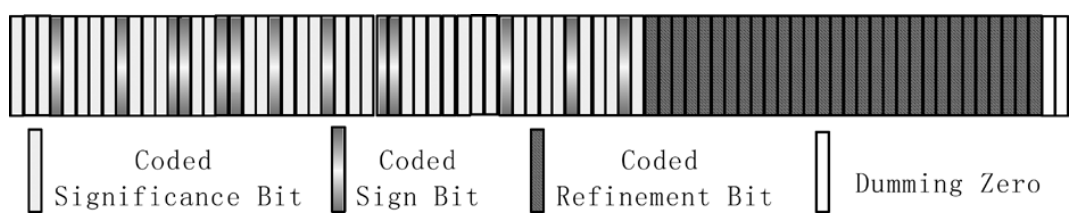

Fig. 2. Syntax of the DU in the original scalable audio bitstream.

partitioning and RVLC [19]. Data partitioning is used to limit the error propagation between different data segments. RVLC is variable length codes (VLC) [15] that can be decoded both in the forward and backward directions. When bit errors occur, the decoder can locate them by comparing the decoding results in the two different directions to limit the scope of error propagation. After ERSAC, the compressed audio stream is passed to the channel-coding module, which performs packetization and error correction using layered product channel codes. Error correction is the key part of our system that enables data recovery from both packet losses and bit errors. After the error control module, the packets are transmitted over the wireless IP network. On the receiver side, the reconstructed packets are directed to the source decoder after channel decoding.

To convey the channel conditions, some related network parameters (e.g., BER, transmission delay and packet loss ratio) from different protocol layers are collected in the network-monitoring module and fed back to the sender. Based on these feedback data, the network status estimation module at the sender chooses a proper model to dynamically estimate network parameters (e.g., the available bandwidth). The target bit allocation module then finds the best source-redundancy tradeoff for error control under the bandwidth constraint.

\section{ERror Resilient SCALAble Audio Coding (ERSAC)}

In our scalable audio codec, the signal is first split into individual time segments, which are filtered by a polyphase quadrature filter $(\mathrm{PQF})$ and down-sampled into four subbands to facilitate scalability in sampling resolution. The modified DCT (MDCT) is then performed on each subband and the resulting MDCT coefficients are weighted by a psychoacoustic mask function [15]. Finally, each weighted subband is encoded into an embedded bitstream using bit-plane coding [30], where each bit plane is coded into one layer or data unit (DU), where DU is the minimal unit for delivery. In Sections IV-A-IV-C, two types of error resilient techniques: data partitioning and RVLC, are described in detail.

\section{A. Data Partitioning}

In bit-plane coding, a coefficient is treated as insignificant if its absolute value is smaller than the threshold (e.g., some power of two). In the beginning, all coefficients are insignificant and the threshold is halved between consecutive bit planes. When a coefficient first becomes significant, the encoder outputs a significance bit plus a sign bit to mark its sign. For coefficients that become significant in previous bit planes, a refinement bit is coded for each of them in the current bit plane.

Each bit plane is coded into a DU, which consists of coded significance/sign bits, followed by coded refinement bits. Fig. 2 illustrates the syntax of DU in the original scalable audio bitstream. Necessary dummy zeros are added for byte-alignment as shown in Fig. 2. The sign bits and refinement bits are not entropy coded, hence bit errors among them will not propagate. In contrast, the significance bits are compressed with VLC, because they are highly correlated. When an error occurs in this portion, it will propagate and the whole DU will be damaged. Multiplexing of DUs worsens this situation because when the decoder detects an error, it does not know the exact error position. Consequently, the whole DU has to be discarded, no matter where the error occurs.

In our proposed ERSAC scheme, we first de-interleave the significance data, the sign data, and the refinement data and put them in three independent partitions. Consequently, any error in the DU is restricted to a particular partition and thus can be isolated. To locate errors among different partitions, the decoder must know the partition boundaries. However, this is impossible 


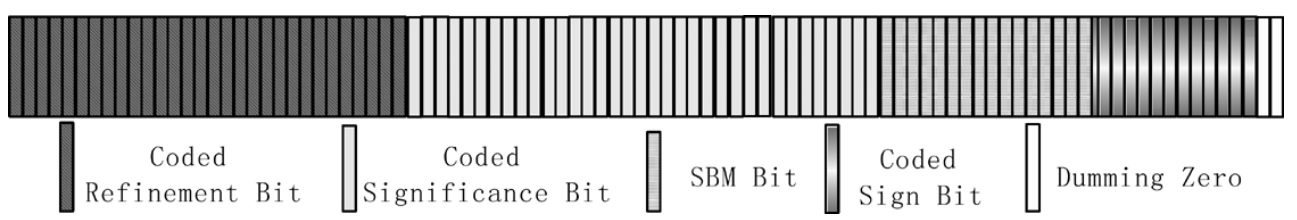

Fig. 3. Syntax of the DU in our proposed ERSAC bitstream.

without a priori side information. Fortunately, bit-plane coding has a property that the number of the refinement bits in the current layer equals that of the significance coefficients in the previous layer. Thus we put the refinement partition in front of other two partitions. In this way, the decoder can deduce the size of the refinement data from the DUs in the previous layer. The size of the significance and sign partitions are unknown before they are coded, we have to introduce significance/sign boundary mark (SBM) to distinguish these two partitions. Because VLC used in our scheme have a finite code tree, we can pick an invalid codeword as the SBM. Moreover, to achieve error robustness, we choose the SBM to be sufficiently far in Hamming distance from other codewords so that it can be detected even when corrupted. The new data structure in ERSAC is depicted in Fig. 3. Notice that the overhead caused in ERSAC is the length of SBM (2-3 bytes), which is nominal when compared to the length of DU.

\section{B. Reversible Variable Length Codes (RVLC)}

RVLC is VLC that can be decoded in both directions. It has received significant attention recently and been applied to error resilient video coding [20], [21]. The two-way decoding property of RVLC generally reduces the coding efficiency. However, there are special types of RVLC that allow two-way decoding while retaining the efficiency of traditional (nonreversible) VLC. Reversible exponential Golomb (Exp-Golomb) codes [22], which are proposed as an extension of the Exp-Golomb codes, belong to this category. Since their length distribution is identical to that in Exp-Golomb codes, they can greatly increase the robustness of the codes without losing coding efficiency.

Like Exp-Golomb codes, reversible Exp-Golomb codes are associated with an order that depends on the source entropy. For binary sources, the optimal choice of the order can be calculated from the probability of zero bits. Based on the order, each codeword includes a variable-length prefix and a fixed-length suffix component. Reversible Exp-Golomb codes are not sensitive to the order and the range of the order is limited. In scalable audio coding, the value of the order is determined by the property of the significance bits in bit-plane coding. In our experiments, the order is set to one in coding the first two bit planes and two in coding the remaining bit planes.

Reversible Exp-Golomb codes are applied to the run lengths of the significance bits in our ERSAC scheme [24]. There is an upper bound for this encoded run length. Once the length of a run exceeds the upper bound, the run is split into multiple runs for independent decoding. There is a tradeoff between error robustness and coding efficiency when choosing the upper bound. It is not desirable to code long run lengths into one codeword because a large error may incur once a bit error happened in a codeword. Thus, it is better to have a small upper bound for the coded run lengths to achieve error resilience. However, splitting long run lengths into smaller ones reduces the coding efficiency.

\section{Error Handling}

Due to data partitioning, the boundary of each partition is known in advance. Consequently, the DU can be easily de-multiplexed into three independent partitions in ERSAC decoding, which limits bit errors to a particular partition. In general, there are two types of bit errors: nonpropagating errors and propagating errors. Nonpropagating errors (e.g., errors in signs and refinement partitions) have limited impairments to the whole partition and they are tolerable by the decoder. In contrast, propagating errors, i.e., errors in significance partitions, have severe impairments and may crash the decoder completely. Hence, they must be detected and located explicitly.

Special error handling is applied to significance partitions, where the data are coded by reversible Exp-Golomb codes for error detection. Because the codewords we use have a finite code tree, some nodes on the tree are invalid and can be served as "traps" to for error detection. When corrupted by an error, the original codeword will turned into an invalid one and can be easily detected in the decoding process. Furthermore, one can also apply sanity checks on the decoded significance bits because the number of the significance bits is known before decoding and the number of binary ones in them must be identical to the number of sign bits. Normally the significance data are decoded both in the forward and backward directions. When an error (e.g., an invalid codeword) is detected, the reversible Exp-Golomb decoder will stop and locate it in both decoding direction. Then, results of the two decoders will be compared and identical portions in the two decoded versions are considered to be correct. By this means, correct bits can be utilized in the subsequent source decoding stage.

\section{ERror Protection With Layered Product Codes}

Error protection in our system is provided by FEC coding that adds parity symbols/packets at the sender to combat packet loss and bit errors during the data transmission process. Layered product codes are designed specifically for this purpose. By applying different product codes to different bitstream layers, more important information in the scalable audio bitstream are highly protected. A product code can be viewed as a two-dimensional code constructed by encoding a rectangle array of information bits with one code along the rows and another one along the columns [11], [12]. The row code is used to combat bit errors and the column code is used to fight packet loss. With product codes, a lost packet registers the loss of both information bits and their corresponding parity 


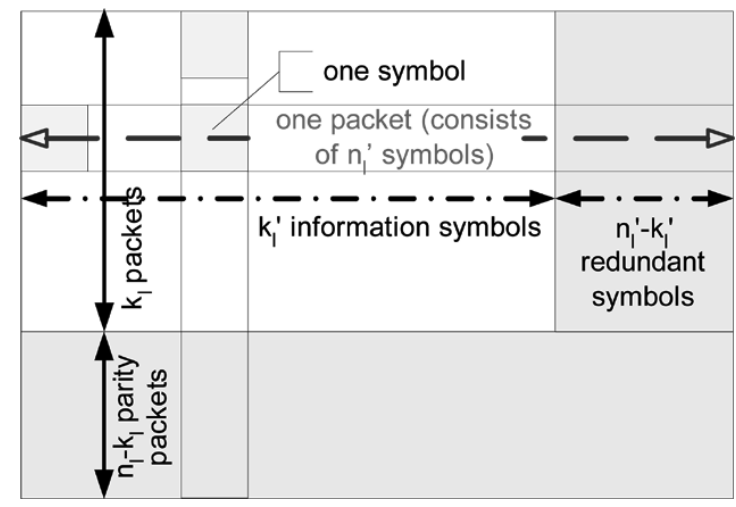

Fig. 4. Structure of product code in a certain layer $l$ with two directional RS codes.

bits generated by the row code in that packet. In this sense, the row code is only helpful in reducing the effect of residual bit errors. Moreover, a cluster of errors within a packet can be treated as a symbol error for the column code. A lost packet also can be regarded as bursty errors in the row direction with known column positions. Therefore the column code can handle not only packet loss but also bit errors.

In this work, Reed-Solomon (RS) codes [23] are used in both the row and column directions, since they are well suited for error protection against bursty packet loss and bit errors. An $(n, k) \mathrm{RS}$ code (with $s$-bit symbols) takes $k$ data symbols and adds $n$ - $k$ parity symbols to make an $n$-symbol codeword, which is capable of correcting up to $t=\lfloor((n-k) / 2)\rfloor$ symbol errors. When the error positions are known, the recoverable number of symbol errors is $t=n-k$. Note that, for a given symbol size $s$, the maximum RS codeword length is $n=2^{s}-1$. One can conceptually shorten RS codes by making a number of data symbols zero at the encoder, not transmitting them and then re-inserting them at the decoder.

Fig. 4 depicts the structure of a single product code used in the $l$ th layer. Note that in this work, the column code is used across packets and the row code is used across symbols within one packet. The $\left(n_{l}, k_{l}\right) \mathrm{RS}$ column code encodes $k_{l}$ information packets across the columns into $n_{l}$ packets. The $\left(n_{l}^{\prime}, k_{l}^{\prime}\right) \mathrm{RS}$ row code encodes $k_{l}^{\prime}$ information symbols into $n_{l}^{\prime}$ symbols within each packet. The symbol size of both $\left(n_{l}, k_{l}\right)$ and $\left(n_{l}^{\prime}, k_{l}^{\prime}\right)$ RS codes is set to 8 for the convenience of accessing information in bytes.

In an $L$-layered audio streaming system, the encoded bitstream is packetized into DUs, and stored on the server side. During audio streaming, several DUs are put into one packet and sent across the wireless IP network. In general, the interdependencies among DUs of different layers can be represented by a directed graph. Fig. 5 shows a typical dependency graph of a group of DUs generated by the scalable audio codec. Each node of the graph corresponds to a DU, and each edge of the graph directed from DU $u^{\prime}$ to $u$ corresponds to a dependence of $u$ on $u^{\prime}$. That is: a DU in one layer depends on all corresponding DUs in preceding layers. Thus the DUs in the first layer are more important than those in the second layer, which are in turn more important than those in the third layer, etc. This sequential dependency calls for UEP in FEC coding.

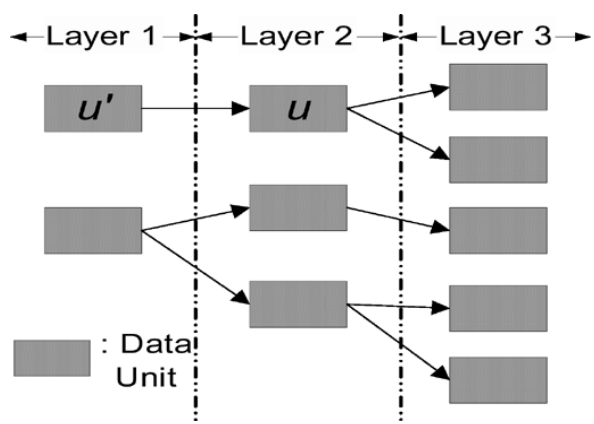

Fig. 5. Typical dependencies among DUs for 3-layered audio bitstream.

Before being delivered to the wireless IP network, DUs are packetized and layered error protection added. Specifically, all $L$ layers of the bitstream are multiplexed into one block of packets (BOP), with $L$ product codes-one for each quality layer, as shown in Fig. 6 on the sender side. Note that since all these $L$ layers construct $n$ packets, the total packet number in the column code for each layer, $n_{l}(1 \leq l \leq L)$, equals $n$. For a given expected transmission rate $R$, the number of packets $n$ in one BOP equals $\left[R / P_{L}\right\rceil$, where $P_{L}$ is the packet size. That is, $n_{l}=n=\left\lceil R / P_{L}\right\rceil$ (for $\left.\forall l: 1 \leq l \leq L\right)$. Information bits in the $l$ th layer are divided into $k_{l}$ rows, each being $k_{l}^{\prime}$ symbols long. The remaining $n-k_{l}$ packets in the BOP are filled with parity bits generated by column channel coding. Within each horizontal packet, the size of the block belonging to layer $l$ is $n_{l}^{\prime}$, with $k_{l}^{\prime}$ information symbols and $n_{l}^{\prime}-k_{l}^{\prime}$ parity symbols generated by row channel coding. Thus, an $\left(n, k_{l}\right) \mathrm{RS}$ code is used along the columns and an $\left(n_{l}^{\prime}, k_{l}^{\prime}\right)$ RS code along the rows. Note that column RS codes for different layers share the same $n$ and $P_{L}=\sum_{i=1}^{L} n_{i}^{\prime}$

The structure of each BOP needs to be transmitted as side information to the receiver, which contains:

- the sequence number of this BOP;

- the number of layers $L$ in this BOP;

- for each layer $l, 1 \leq l \leq L$ :

- the number of packets, $k_{l}$, that contain the information data for layer $l$;

- the number of information symbols $k_{l}^{\prime}$ that layer $l$ occupies in each packet;

- the number of redundant symbols $n_{l}^{\prime}-k_{l}^{\prime}$ in each packet for layer $l$.

Since the size of this side information is quite small, we assume that it can be successfully transmitted with powerful FEC and automatic repeat request (ARQ) techniques. Then, the total transmission rate $R$ with our packetization scheme can be calculated as

$$
R=\sum_{l=1}^{L} \frac{n \times n_{l}^{\prime}}{k_{l} \times k_{l}^{\prime}} R_{l}^{\prime}
$$

where $R_{l}$ is the information rate for the $l$ th layer (the rate for specifying side information is ignored).

The above layered product codes facilitate UEP among different layers for scalable audio. One can adjust the rates of both row and column codes at each layer according to the network condition. 


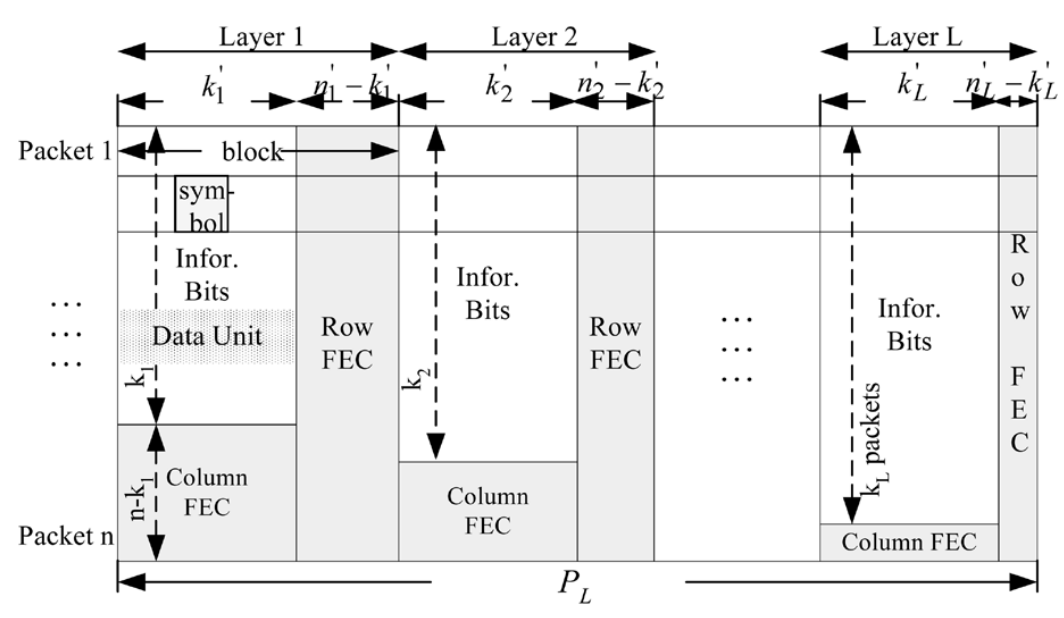

Fig. 6. The illustrative diagram for the BOP of our packetization scheme.

\section{OPTIMAL BIT ALLOCATION FOR ERROR CONTROL}

\section{A. Bit Allocation Between Source and Channel Protection}

As stated in Section II, the network status is monitored on the server side. The main issue for the server is how to efficiently utilize the limited network resource under varying network conditions. It is known that under a given channel condition, additional FEC increases error robustness and in turn reduces the available bit rate for source coding. Thus, there is a tradeoff between source coding and FEC [17], [25], [26]. Based on the wireless IP channel characteristics (e.g., possible packet losses and residual bit errors), we search for the best tradeoff in error control via optimal bit allocation to mitigate the effect of packet losses and bit errors. The aim of bit allocation is to minimize the expected end-to-end distortion by determining the optimal source coding rates $R_{1}, \ldots, R_{L}$, column code rate vector $\bar{k}$, and the row code rate vectors $\bar{n}^{\prime}$ and $\bar{k}^{\prime}$ for different layers under a given target bit rate constraint.

In a wireless network, the end-to-end distortion $D$ is composed of the source quantization distortion $D_{s}$ and the channel error distortion $D_{c}$. Without loss of generality, it is assumed that the source quantization error is orthogonal to the channel error. Then, the end-to-end distortion is represented as

$$
D(R)=D_{s}\left(R_{s}\right)+D_{c}\left(R_{c}, R_{s}\right)
$$

where $D_{s}\left(R_{s}\right)$ is the source rate-distortion curve and $D_{c}\left(R_{c}, R_{s}\right)$ represents a channel rate-distortion curve reflecting channel distortion as a function of the channel coding rate $R_{c}$ and source coding rate $R_{s}$. For a given target rate $R$, we fix packet length $P_{L}$, then the number of packets $n=\left\lceil R / P_{L}\right\rceil$ is known. Define $\bar{k}=\left[k_{1}, \ldots, k_{L}\right] ; \bar{n}^{\prime}=\left[n_{1}^{\prime}, \ldots n_{L}^{\prime}\right]$; $\bar{k}^{\prime}=\left[k_{1}^{\prime}, \ldots, k_{L}^{\prime}\right]$, then the optimal bit allocation problem can be formulated as

$$
\begin{aligned}
\min _{\left\{R_{1}, \ldots, R_{L}, \bar{k}, \bar{n}^{\prime}, \bar{k}^{\prime}\right\}} & D(R)=\min \left(D_{s}\left(R_{s}\right)+D_{c}\left(R_{c}, R_{s}\right)\right) \\
\text { subject to } & R_{s}+R_{c} \leq R
\end{aligned}
$$

where $D_{s}\left(R_{s}\right)=A 2^{-2 R_{s}}$ [4] with $A$ being a constant, $R_{s}=$ $\sum_{i=1}^{L} R_{i}$ and $R_{c}=\sum_{i=1}^{L}\left(\left(n / k_{i}\right)\left(n_{i}^{\prime} / k_{i}^{\prime}\right)-1\right) R_{i}$.

The unknown variables in the above formulation are $R_{1}, \ldots, R_{L}$ and vectors $\bar{k}, \bar{n}^{\prime}$, and $\bar{k}^{\prime}$; the constraints are
$R=\sum_{l=1}^{L}\left(n \times n_{l}^{\prime} / k_{l} \times k_{l}^{\prime}\right) R_{l}$ and $P_{L}=\sum_{i=1}^{L} n_{i}^{\prime}$. This minimization problem is very different from standard bit allocation problems because: 1 ) the expression for $D(R)$ cannot be split into a sum of terms, each depending on a single unknown variable and 2) the total rate $R$ is not a linear function of the unknown variables.

In the following, we first give an analytical expression of $D_{c}\left(R_{c}, R_{s}\right)$ (hence the end-to-end distortion $D(R)$ ), we then use an iterative procedure to search for the optimal solution to our bit allocation problem. Details of the analysis algorithm appear in Appendix.

\section{B. Derivation of the End-to-End Distortion Metric}

We observe the sequential dependency among DUs in different layers in the source bitstream when deriving $D_{c}\left(R_{c}, R_{s}\right)$. Depending on the number of lost packets, we first check if the DUs in the first layer could be decoded, then see if the DUs in the first and second layers could be decoded, etc. In the meanwhile, row channel codes are primarily viewed as means of correcting bit errors in horizontal blocks within layers.

Proposition: Let the column RS codes for the $L$ layers be parameterized by $\left(n, k_{1}\right),\left(n, k_{2}\right), \ldots,\left(n, k_{l}\right)$ with $k_{1} \leq k_{2} \leq$ $\cdots \leq k_{L}$. Depending on the number of lost packets $r(0 \leq r \leq$ $n$ ), define the first number of layers $c(r)$ that can be correctly decoded as

$$
\left.c(r)=\arg \max _{j=0, \ldots, L}\left\{\left(n-k_{j}\right)>r\right)\right\} .
$$

Then the expected end-to-end distortion can be described as

$$
\begin{aligned}
D(R)= & D_{s}\left(R_{s}\right)+D_{c}\left(R_{c}, R_{s}\right) \\
= & A 2^{-2 R_{s}} \\
& +\sum_{r=0}^{n}\left\{P(r, n) \sum_{l=1}^{L}[B(l, r)\right. \\
& \left.\left.\times\left(\prod_{j=1}^{l-1} P_{\mathrm{dep}}(j, c(r), r)\right) \Delta D_{l}\right]\right\}
\end{aligned}
$$

where $P(r, n)$ is the probability of losing $r$ out of $n$ packets, $B(l, r)$ is the expected number of the erroneous blocks in the $l$ th layer when the number of lost packets is $r, P_{\mathrm{dep}}(j, c(r), r)$ 
is the average probability of a block in the $j$ th layer being correctly decodable if $c(r)$ layers are correctly decoded with $r$ lost packets, and $\Delta D_{l}$ denotes the distortion caused by one lost block in the $l$ th layer, which renders all remaining blocks in the same packet useless. It can be seen from the above equation that the end-to-end distortion depends on $P(r, n), P_{\mathrm{dep}}(j, c(r), r)$ and $B(l, r)$, which are all related to network conditions (i.e., average packet loss ratio and average residual BER). That is to say, it would be essential that the bit allocation scheme can adapt to varying network conditions.

$P(r, n)$ is the probability that $r$ out of $n$ packets are lost. As stated above, we use the 2-state Markov model to estimate network packet loss status. This model is determined by the distribution of loss-free intervals (gap). Let gap length $v$ be the event that after a lost packet, $v-1$ packets are received and then another packet is lost. The gap density function $g(v)$ gives the probability of gap length $v$, i.e., $g(\nu)=\operatorname{Pr}\left(1^{v-1} 0 \mid 0\right)$. The gap distribution function $G(v)$ is the probability that the gap length is greater than $v-1$, i.e., $G(v)=\operatorname{Pr}\left(I^{v-1} \mid 0\right)$. They can be derived as

$$
\begin{aligned}
g(v) & = \begin{cases}1-p, & \text { for } v=1 \\
p(1-q)^{v-2} q, & \text { for } v>1\end{cases} \\
G(v) & = \begin{cases}1, & \text { for } v=1 \\
p(1-q)^{v-2}, & \text { for } v>1\end{cases}
\end{aligned}
$$

where $p$ and $q$ are the transition probabilities defined in Section II-A. Let $R(m, n)$ be the probability of $m-1$ packet losses within the next $n-1$ packets followed by a lost packet. It can be calculated using recurrence as follows:

$R(m, n)= \begin{cases}G(n), & \text { for } m=1 \\ \sum_{v=1}^{n-m+1} g(v) R(m-1, n-v), & \text { for } 2 \leq m \leq n .\end{cases}$

Then the probability of $m$ lost packets within $n$ packets is

$$
P(m, n)=\sum_{v=1}^{n-m+1} P_{B} G(v) R(m, n-v+1), \quad \text { for } 1 \leq m \leq n
$$

where $P_{B}$ is the average of packet-loss probability.

Lemma 1: If the number of lost packets is $r$ and $t_{i}=\left\lfloor n-r-k_{i}\right\rfloor / 2$, the dependent probability of the $i$ th layer to the $l$ th layer, $P_{\mathrm{dep}}(i, c(r), r)$ can be represented as shown in (11), at the bottom of the page, where $t_{i}$ is the number of the redundancy blocks available for bit errors, $P_{G L}(i, r)$ is the accurate probability of layer $i$, which means that all the bits in layer $i$ can be correctly decoded, and $P_{g r}(i, r)$ is the accurate probability of one block in layer $i$, which can be further calculated as

$$
P_{g r}(i, r)=\sum_{m=0}^{t_{i}^{\prime}} C_{n_{l}^{\prime}}^{m}\left(P_{s}\right)^{m}\left(1-P_{s}\right)^{n_{l}^{\prime}-m}
$$

where $P_{s}$ is the corrupted probability of a symbol and $t_{i}^{\prime}$ equal $\left\lfloor n_{i}^{\prime}-k_{i}^{\prime}\right\rfloor / 2$.

Lemma 2: If the number of lost packets is $r$ and $t_{l}=\lfloor n-$ $\left.r-k_{l}\right\rfloor / 2$, the expected number of erroneous blocks $B(l, r)$ is given by (13), shown at the bottom of the page.

More details on these two lemmas are given in the Appendix.

In this section, the overall rate-distortion relation is analyzed. Therefore the bit allocation can be performed by searching the optimal rate sets of each layer [32].

\section{Simulation Results}

Simulations are performed to demonstrate the effectiveness of our proposed error robustness tools for audio streaming. The purpose of this simulation is to show: 1) our proposed ERSAC technology provides error resilience in the source audio coding level and 2) the layered-product-code error protection scheme provides error robustness in the transport level to combat the packet losses and bit errors occurred in the wireless networks simultaneously.

\section{A. Simulation Setup and Parameters}

In our simulation, a single-cell CDMA system is considered with several mobile stations in a wireless flat fading channel. The transmitted data at mobile station is first encoded using a rate $1 / 2$ convolutional code. The coded data is interleaved, segmented, and mapped to the physical channel after variablelength spreading. The radio transmission parameters and propagation models, from outdoor to indoor and pedestrian environments, are set according to the IMT-2000 evaluation methodology [28]. Both large-scale path loss and small-scale fading are experienced in the channel. In our implementation, the Jakes model is adopted to simulate the fading channel [5]. A standard RAKE receiver is used at base station. For simplicity, we assume the complex conjugate channel coefficients are available at the receiver RAKE. The application layer data is packetized and transported in UDP packets with the packet size of 576 bytes. The UDP packet can be further segmented into several RLC frames. The maximal number of retransmission times for a RLC frame varies from 0 to 3. Other parameters are tabulated in Table I.

$$
P_{\mathrm{dep}}(i, c(r), r)= \begin{cases}P_{G L}(i, r)+\left(1-P_{G L}(i, r)\right) \frac{\left(n-r-t_{i}-1\right)}{n}, & \text { if } i \leq c(r) \\ P_{g r}(i, r) \frac{n-r}{n}, & \text { if } c(r)<i \leq L\end{cases}
$$

$$
B(l, r)= \begin{cases}\left.\left(1-P_{G L}(l, r)\right)(r+t+1)+(n-r-t-1) P_{g r}(l, r)\right), & l \leq c(r) \\ (n-r)\left(1-P_{g r}(l, r)\right)+r, & l=c(r)+1 \\ (n-r)\left(1-P_{g r}(l, r)\right), & c(r)+1<l \leq L .\end{cases}
$$




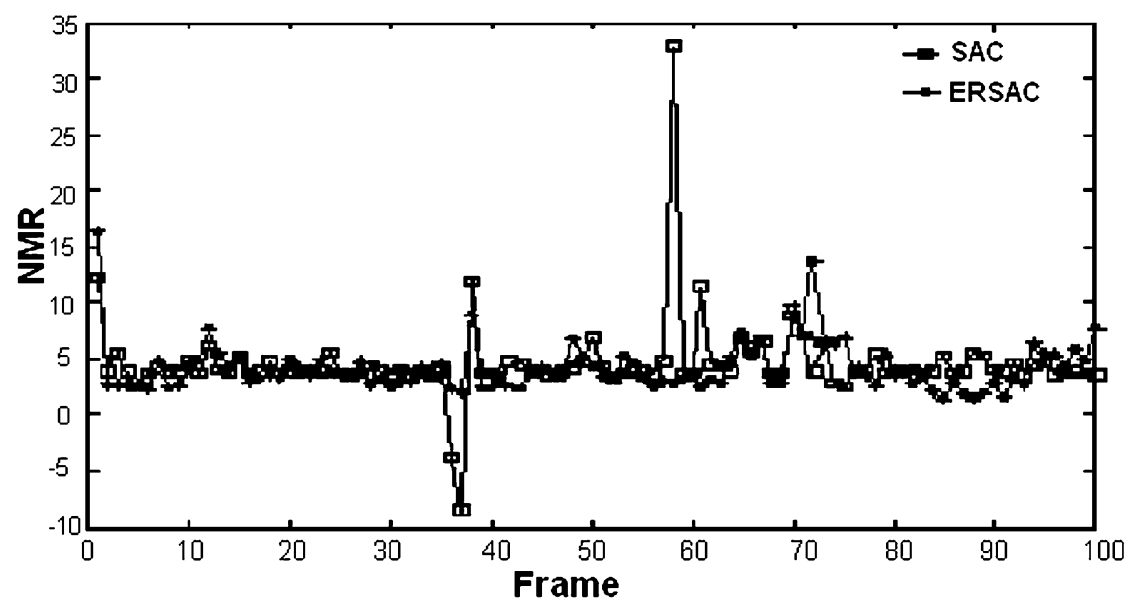

(a)

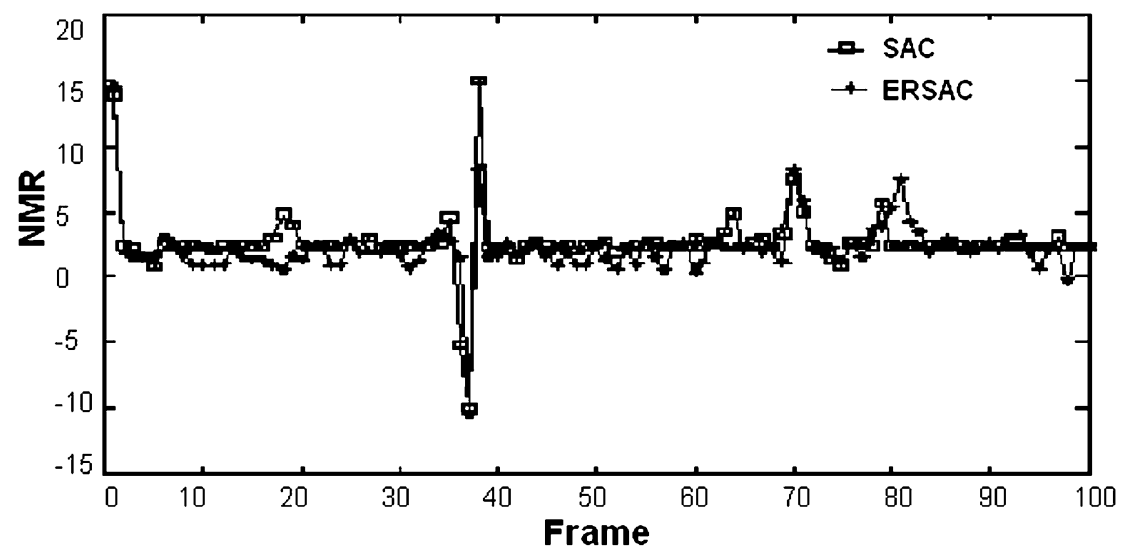

(b)

Fig. 7. NMRs of SAC and ERSAC for the audio clip horn $23 \_2$ with different bit error ratios: (a) 10e-3 and (b) 10e-4. The bit rate for horn23_2 is 64 kbps.

TABLE I

SIMULATION PARAMETERS

\begin{tabular}{c|c}
\hline Chip Rate & $11.0593 \mathrm{Mchips} / \mathrm{sec}$ \\
\hline Carrier Frequency & $1900 \mathrm{MHz}$ \\
\hline Transmit Power adjust step size & $0.25 \mathrm{~dB}-1.5 \mathrm{~dB}$ \\
\hline Power Amplifier & Noise figure $=5 \mathrm{~dB}$ \\
\hline Uplink Transmission Power Constraint & $20 \mathrm{~mW}$ \\
\hline Cell range & $50 \mathrm{~m}-300 \mathrm{~m}$ \\
\hline Moving speed & $24 \mathrm{~km} / 30 \mathrm{~km} / \mathrm{h}$ \\
\hline RLC-PDU (Protocol Data Unit) & $08 \mathrm{bits}$ \\
\hline CRC bits & $0 / 1 / 2 / 3$ \\
\hline Maximal retransmission times & 576 bytes \\
\hline UDP packet size & Low error case: $1.5 * 10 \mathrm{e}-5 \sim 2.5 * 10 \mathrm{e}-5$ \\
Residual BER target & High error case: $2 * 10 \mathrm{e}-3 \sim 3 * 10 \mathrm{e}-3$ \\
\hline
\end{tabular}

\section{B. Performance Evaluation of Error Resilience}

Extensive simulations are carried out to test the performance of our proposed ERSAC scheme. The MPEG-4 standard audio clips horn23_2,trpt21_2 and vioo10_2 are used for testing. The scalable audio coder encodes each audio clip at a fixed rate of 64 kbps. In our experiments, we simulate a wireless network environment using a Rayleigh model [5] with the following parameters: $E b / N_{o}=22 \mathrm{db}, \mathrm{BER}=1 e-3$, and $E b / N_{o}=32 \mathrm{db}$, $\mathrm{BER}=1 e-4$. The walking velocity for common walkers is $3 \mathrm{~km} / \mathrm{h}$. Such conditions are obtained after passing through physical layer and data link layer of wireless networks and are typical error conditions that mobile applications have. We assume that there is no channel coding applied to the scalable bitstream and the header has been well protected. We illustrate the quality of the decoded audio through the objective measurements of the noise-mask-ratio (NMR) [27]. For a given band, NMR is the ratio of the noise energy to the masking threshold. It may equivalently be viewed as a weighted squared error measure, $w_{i} d_{i}$, where $d_{i}$ is the quantization noise energy (squared error) in band $i$ and the weight, $w_{i}$, is chosen as the inverse of the masking threshold in that band. It can be seen that a lower value of NMR shows a better audio quality. In the simulations, our ERSAC scheme is compared to the original scalable audio coding scheme, referred to as SAC for simplicity.

The NMRs of SAC and ERSAC under the BER of 10e-3 and 10e-4 are shown in Fig. 7(a) and (b), respectively. There are some bursty peaks on curves for SAC, showing the occurrence of bit errors; while there are almost no bursty peaks on the curves for ERSAC. This proves that ERSAC indeed improves the error resilience of scalable audio coding and is more immune to bit errors than SAC. The numerical NMR results for streaming of audio clips honrn23_2, trpt21 and vioo10_2 under constant network bandwidth (64 kbps) and BER of 10e-3 and 10e-4 are shown in Table II. It is obvious that ERSAC outperforms SAC as it provides lower average NMR values for all clips and BERs.

Subjective tests have also been conducted for the conditions listed in Table II. Listeners perceive a better quality of the delivered audio given by ERSAC, while annoying artifacts are audible from SAC. This further verified that the proposed 
TABLE II

AVERAGE NMR RESUlTS FOR MULTIPLE AUDIO CliPS WITH DIFFERENT BERs

\begin{tabular}{|c|c|c|c|c|c|c|}
\hline \multirow{2}{*}{$\begin{array}{l}\text { Audio } \\
\text { Clips }\end{array}$} & \multicolumn{2}{|c|}{ Horn23_2 } & \multicolumn{2}{|c|}{ Trpt21_2 } & \multicolumn{2}{|c|}{ Vioo10_2 } \\
\hline & BER $10^{-3}$ & BER $10 \mathrm{e}^{-4}$ & BER $10 \mathrm{e}^{-3}$ & BER $10 \mathrm{e}^{-4}$ & BER $10 \mathrm{e}^{-3}$ & BER $10 \mathrm{e}^{-4}$ \\
\hline SAC & 4.5713 & 2.6185 & 4.4139 & 2.7738 & 4.4192 & 3.0124 \\
\hline ERSAC & 4.2045 & 2.1698 & 4.1738 & 2.1050 & 4.0264 & 2.1757 \\
\hline
\end{tabular}

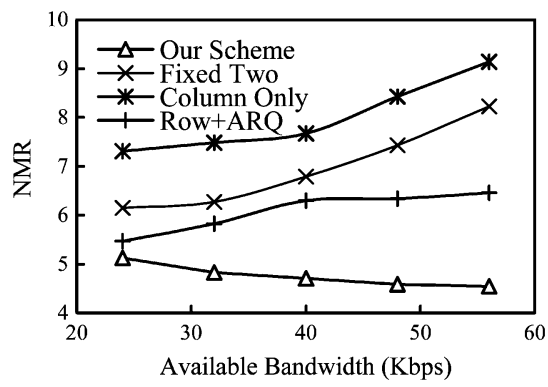

(a)

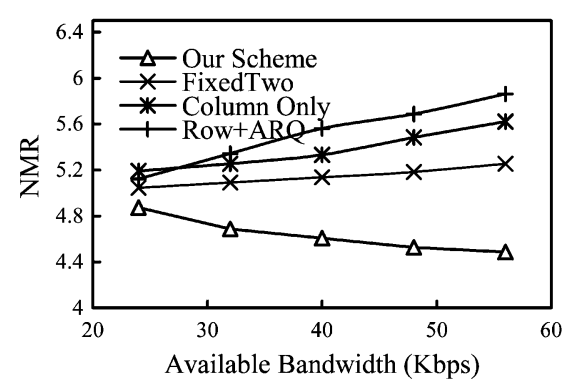

(b)

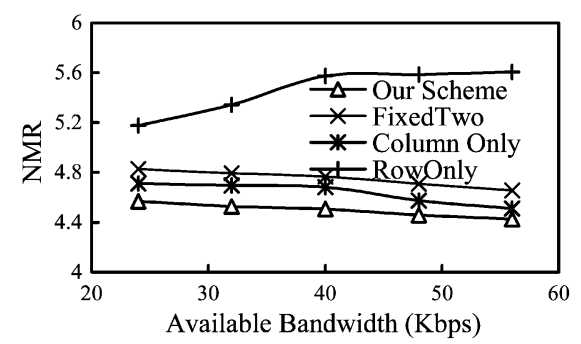

(c)

Fig. 8. Average NMR for horn23_2 using different protection schemes under different bit rates. (a) $15 \%$ packet loss ratio and BER $2.532 \times 10 \mathrm{e}-3$. (b) $5 \%$ packet loss ratio and BER $2.532 \times 10 \mathrm{e}-3$. (c) $0.5 \%$ packet loss ratio and BER $2.532 \times 10 \mathrm{e}-3$.

ERSAC scheme with data partition and RVLC greatly improves the quality of wireless audio.

\section{Performance of Our Adaptive Error Control Scheme}

This simulation is to demonstrate effectiveness of our proposed channel-adaptive error protection scheme for scalable audio streaming. In this simulation we tested four schemes: 1) fixed UEP at two directions (20\% for the first layer and $10 \%$ for other layers in the column channel-code, $10 \%$ for the first layer and 5\% for other layers in the row channel-code); 2) fixed column channel code only ( $20 \%$ for the first layer and $10 \%$ for the rest of the layers); 3) fixed row channel code with packet level ARQ (10\% for the first layer and 5\% for the rest of the layers) - note that the retransmission times is limited to one to ensure delay requirements; 4) our proposed channel-adaptive layered product code-based error protection scheme.

In all the tested cases, the MPEG-4 standard audio clip, horn_23_2, is used for testing. The scalable audio coder encodes the audio signals at a coding rate of $64 \mathrm{kbps}$. All simulations are performed with the packet loss ratios of the network varying from $0.5 \%$ to $15 \%$. We test these schemes on channels with different residual BERs. More specifically, we classify these channels into two types: high residual BER channel with average value of $2.532 \times 10^{-3}$ and low residual BER channel with average value of $1.8 \times 10^{-5}$. The total available bandwidth is the same for all the protection schemes in all cases. All results are averaged over 30 Monte Carlo simulations [29].

Figs. 8 and 9 show average NMR results obtained by using different protection schemes at different bit rates under various channel conditions. It can be seen from these two figures that our proposed product code structure obtains better results than the other three tested schemes in wireless networks.

Notice that for the scalable bitstream, bits in higher layers have dependency on those in lower layers. Thus, with more available bandwidth, the more we need to protect lower layer bits. In our error protection scheme, the protection degree for the lower layers can be increased according to the source rate-distortion function. On the contrary, for the fixed error protection scheme, the protection degree for each layer is fixed. Therefore, the correctly decodable probability of bits in higher layers decreased as the available bandwidth increases. Thus, the performance of the fixed schemes does not improve with the increase of the available bandwidth. This can be observed in both Figs. 8 and 9 .

Another interesting phenomenon we observe is that the performance of the fixed error protection schemes becomes worse in bad channel conditions [see Figs. 8 and 9(a)]. Specially, it can be seen that when the error scheme is too weak to recover the corrupted information, its performance may even decreased with the increase of the available bandwidth. On the other hand, when higher protection is adopted, its performance becomes better with the increase of the available bandwidth. Among the three compared error protection schemes, it can be observed that ARQ is preferable for channels with high loss packet ratio [see Figs. 8(a) and 9(a)]; while the FEC technology is preferable for channels with lower loss packet ratio [see Fig. 8(b) and (c) and Fig. 9(b) and (c)]. Finally, in high BER scenarios, row channel codes are very helpful in combating bit errors as they present a clean "channel" for column channel codes. But in low BER scenarios, using row channel codes is inefficient. 


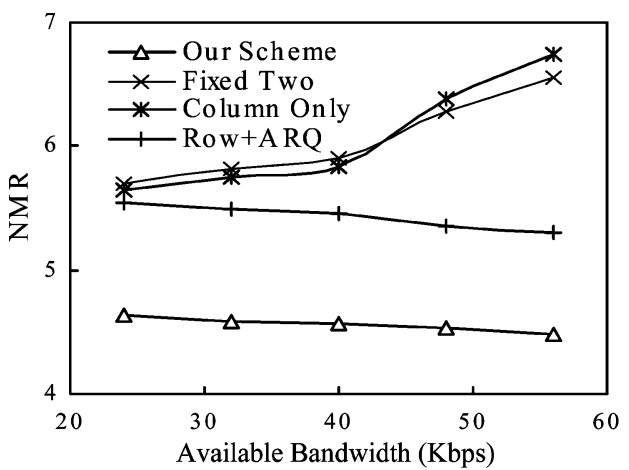

(a)

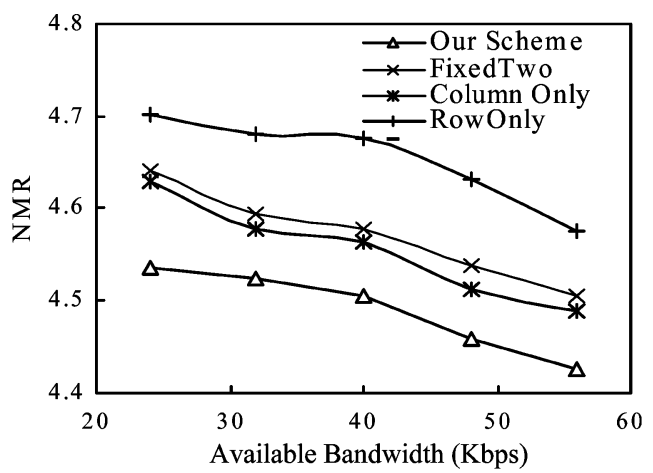

(b)

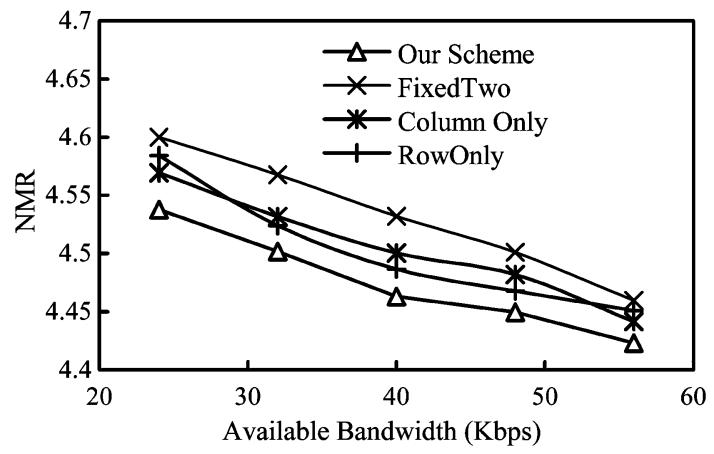

(c)

Fig. 9. Average NMR for horn 23 _2 using different protection schemes under different bit rates. (a) $15 \%$ packet loss ratio and BER $1.8 \times 10 \mathrm{e}-5 .(\mathrm{b}) 5 \%$ packet loss ratio and BER $1.8 \times 10 \mathrm{e}-5$. (c) $0.5 \%$ packet loss ratio and BER $1.8 \times 10 \mathrm{e}-5$.

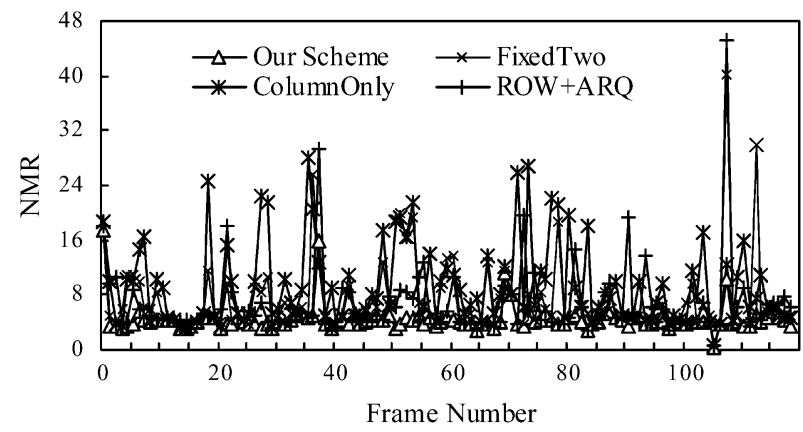

(a)

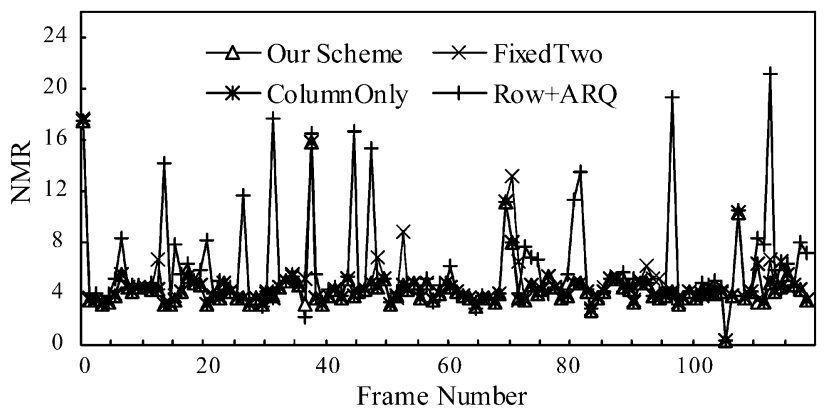

(b)

Fig. 10. NMR comparison results for horn $23 \_2$ using different protection schemes at $56 \mathrm{kbps}$ bit rate under high BER channels. (a) $15 \%$ packet loss ratio. (b) $0.5 \%$ packet loss ratio.

Figs. 10 and 11 show the average NMR results obtained by using different protection schemes at $56 \mathrm{kbps}$ bit rate under different channel conditions. It can be seen from those figures that

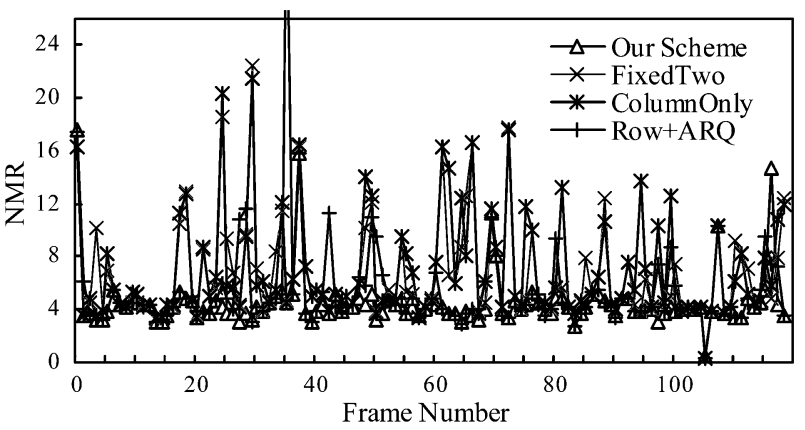

(a)

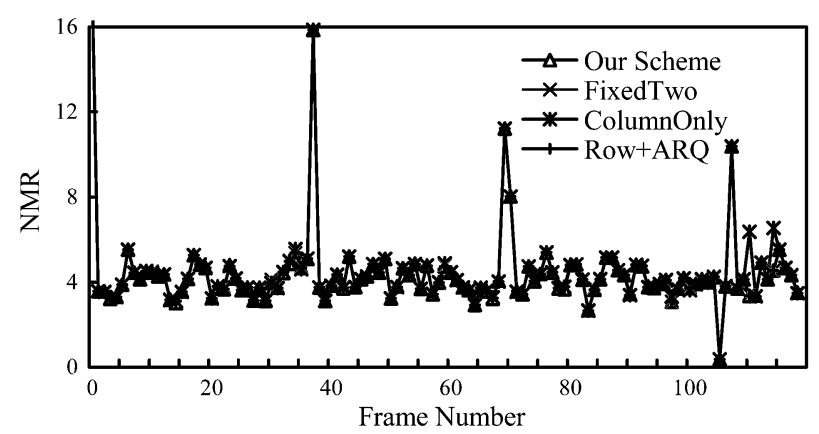

(b)

Fig. 11. The NMR comparison results for horn23_2 using different protection schemes at $56 \mathrm{kbps}$ bit rate under low BER channel. (a) $15 \%$ packet loss ratio. (b) $0.5 \%$ packet loss ratio.

our proposed approach obtains better results than the other three tested schemes. Specifically, with $15 \%$ packet loss ratio [see Figs. 10(a) and 11(a)], all the other three schemes show severe 
noise artifacts, which are not acceptable to the listeners. Moreover, the scheme (4) with ARQ is not suitable for channel with low loss packet ratios [see Fig. 10(b)].

In summary, the simulation results presented in this section conclusively demonstrate that: 1) ERSAC provides error resilient in the audio codec level and 2) our proposed error control scheme provides error robust in application transport level by correcting the packet losses and bit errors simultaneously.

\section{CONCLUSIONS}

This paper presented an ERSAC approach for providing error resilience in the audio codec level, while proposing a novel layered product code based error control approach for providing error robustness in transport level by protecting the packet losses and residual bit errors simultaneously. In our proposed approach, we performed data partition and reversible variable length coding to enhance the resilience of scalable audio. Considering the characteristic of scalable audio, unequal row channel code and unequal column code are adopted for different layers of the scalable audio bitstream. Moreover, rate-distortion based bit allocation is proposed to determine the channel coding rate and source coding rate so as to minimize the expected end-to-end distortion. Simulation results show that ERSAC is very robust to the bit errors, and our proposed error control scheme outperforms other schemes under different network conditions.

This paper presents an error robust scheme for scalable audio streaming over wireless IP networks. Our proposed error protection scheme applies equally to other compressed media (e.g., video) with layered structure.

\section{APPENDIX}

Lemma 1: If the number of lost packets is $r$ and $t_{i}=\left\lfloor n-r-k_{i}\right\rfloor / 2$, the dependent probability of the $i$ th layer to the $l$ th layer, $P_{\text {dep }}(i, c(r), r)$ (for $i<l$ ), can be represented as shown in (a.1), at the bottom of the page, where $P_{G L}(i, r)$ is the accurate probability of layer $i$, which means that all the bits in layer $i$ can be successfully recovered, and $P_{g r}(i, r)$ is the correct probability of one block in layer $i$, which can be further represented as

$$
P_{g r}(i, r)=\sum_{m=0}^{t_{i}^{\prime}} C_{n_{l}^{\prime}}^{m}\left(P_{s}\right)^{m}\left(1-P_{s}\right)^{n_{l}^{\prime}-m}
$$

where $P_{s}$ is the corrupted probability of a symbol and $t_{i}^{\prime}$ equal $\left\lfloor n_{i}^{\prime}-k_{i}^{\prime}\right\rfloor / 2$. For the layer not higher than $c(r)(i \leq c(r))$, the accurate probability of layer $i, P_{G L}(i, r)$ comes from two parts. One is the case in which the number of the corrupted blocks is less than $t_{i}$, which equals to

$$
\sum_{m_{e}=0}^{t_{i}} C_{n-r}^{m_{e}}\left(1-P_{g r}(i, r)\right)^{m_{e}}\left(P_{g r}(i, r)\right)^{n-r-m_{e}} \text {. }
$$

Without loss of generality, it is assumed that the blocks corrupted by the bit errors are randomized.

The second case considers the number of the corrupted blocks is more than $t_{i}$. However, even in this case, the bit errors still can be recovered because not all the errors lie at the same symbolcolumn.

Denote $m_{c}$ as the number of corrupted blocks in the following discussion. Let us define $P_{g c}\left(m_{e}, i, r\right)$ as the accurate probability of one symbol-column under the condition that there are $m_{e}$ corrupted blocks in layer $i$ and $r$ lost packets. Thus, the second part of $P_{G L}(i, r)$ is formulated as

$$
\begin{aligned}
\sum_{m_{e}=t_{i}+1}^{n-r} C_{n-r}^{m_{e}}(1 & \left.-P_{g r}(i, r)\right)^{m_{e}} \\
& \times\left(P_{g r}(i, r)\right)^{n-r-m_{e}}\left(P_{g c}\left(m_{e}, i, r\right)\right)^{n_{l}^{\prime}}
\end{aligned}
$$

where

$$
P_{g c}\left(m_{e}, i, r\right)=\sum_{x=0}^{t} C_{m_{e}}^{x}\left(P_{s}\right)^{x}\left(1-P_{s}\right)^{m_{e}-x} .
$$

Combining the expressions (a.3) and (a.4), the accurate probability of layer $j$, can be represented as

$$
\begin{aligned}
P_{G L}(i, r)= & \sum_{m_{e}=0}^{t_{i}} C_{n-r}^{m_{e}}\left(1-P_{g r}(i, r)\right)^{m_{e}}\left(P_{g r}(i, r)\right)^{n-r-m_{e}} \\
& +\sum_{m_{e}=t_{i}+1}^{n-r} C_{n-r}^{m_{e}}\left(1-P_{g r}(i, r)\right)^{m_{e}} \\
& \times\left(P_{g r}(i, r)\right)^{n-r-m_{e}}\left(P_{g c}\left(m_{e}, i\right)\right)^{n_{i}} .
\end{aligned}
$$

In the case that some blocks are corrupted by the bit errors, it does not mean that all the data in the higher layers are disabled. Let us discuss the probability of the higher layers relying on layer $i$, which can be approximately represented as shown in (a.7), at the bottom of the page The second part of the (a.7) is equal to

$$
\frac{1}{n}\left(1-P_{G L}(i, r)\right) \times E\left(m_{e}>t_{i}+1\right)
$$

$$
P_{\mathrm{dep}}(i, c(r), r)= \begin{cases}P_{G L}(i, r)+\left(1-P_{G L}(i, r)\right) \frac{\left(n-r-t_{i}-1\right)}{n}, & \text { if } i \leq c(r) \\ P_{g r}(i, r) \frac{n-r}{n}, & \text { if } c(r)<i \leq L\end{cases}
$$

$$
\begin{aligned}
& \sum_{m_{e}=t_{i}+1}^{n-r} C_{n-r}^{m_{e}}\left(1-P_{g r}(i, r)\right)^{m_{e}}\left(P_{g r}(i, r)\right)^{n-r-m_{e}}\left(1-\left(P_{g c}\left(m_{e}, l\right)\right)^{n_{i}^{\prime}}\right) \frac{n-r-m_{e}}{n} \\
& =\left(1-P_{G L}(i, r)\right) \frac{n-r}{n}-\frac{1}{n}\left(1-P_{G L}(i, r)\right) \\
& \quad \times \sum_{m_{e}=t_{i}+1}^{n-r} \frac{C_{n-r}^{m_{e}}\left(1-P_{g r}(i, r)\right)^{m_{e}}\left(P_{g r}(i, r)\right)^{n-r-m_{e}}\left(P_{g c}\left(m_{e}, i, r\right)\right)^{n_{l}^{\prime}}}{1-P_{G L}(i, r)} m_{e} .
\end{aligned}
$$




$$
B(l, r)= \begin{cases}\left(1-P_{G L}(l, r)\right)\left((r+t+1)+(n-r-t-1) P_{g r}(l, r)\right) & l \leq c(r) \\ (n-r)\left(1-P_{g r}(l, r)\right)+r & l=c(r)+1 \\ (n-r)\left(1-P_{g r}(l, r)\right) & c(r)+1<l \leq L .\end{cases}
$$

where $E\left(^{*}\right)$ is the expected number of the corrupted blocks by the bit errors on the condition that $m_{e}$ of $n$ is larger than $t+1$.

$E\left({ }^{*}\right)$ in (a.8) also is

$E\left(m_{e}>t+1\right)=(t+1)$

$+E$ (number of corrupted block within $n-t-1-r)$.

Assuming each event of such block is corrupted is independent, the second part of (a.9) can be simplified to

$$
\left(n-r-t_{i}-1\right)\left(1-P_{g r}(i, r)\right) .
$$

Replace (a.8)-(a.10) to (a.7), we get

$$
\left(1-P_{G L}(i, r)\right) \frac{\left(n-r-t_{i}-1\right)}{n} P_{g r}(i, r) .
$$

The intuitive meaning from the above expressions is that, the probability affecting the block in the higher following layers is not only related to the total number of the erroneous blocks in layer $i, r+t_{i}+1$, but also related to the accurate probability within one block $P_{g r}(i, r)$, which deals with the bit errors within the block.

On the other hand, for layer $i, l>c(r)$, the lost blocks cannot be recovered. Therefore only those blocks immune from the bit errors make latter corresponding data decodable. This probability is formulated as

$$
\sum_{m_{e}=0}^{n-r} C_{n-r}^{m_{e}}\left(1-P_{g r}(i, r)\right)^{m_{e}}\left(P_{g r}(i, r)\right)^{n-r-m_{e}} \frac{n-r-m_{e}}{n}
$$

which can be simplified as $P_{g r}(i, r)(n-r / n)$.

Lemma 2: The expected number of erroneous blocks, $B(l, r)$, is given by (a.13), shown at the top of the page. For the first case, $l \leq c(r)$, the number of the erroneous blocks can be calculated by

$$
\begin{aligned}
\sum_{m_{e}=t+1}^{n-r} C_{n-r}^{m_{e}}(1- & \left.P_{g r}(l, r)\right)^{m_{e}}\left(P_{g r}(l, r)\right)^{n-r-m_{e}} \\
& \times\left(1-\left(P_{g c}\left(m_{e}, l, r\right)\right)^{n_{l}}\right)\left(r+m_{e}\right) .
\end{aligned}
$$

Similar to the analysis of (a.7), (a.14) equals to

$$
\left(1-P_{G L}(l, r)\right)\left((r+t+1)+(n-r-t-1)\left(1-P_{g r}(l, r)\right) .\right.
$$

Note that for the layer, $l \geq(c(r)+1)$, it is further divided into case 2 and case 3 . Considering the dependency relation with higher layers and lower layers, the number of erroneous blocks in layer $l, l>(i+1)$, do not include the lost blocks because they have been counted in the end-to-end distortion in the lower layers.

\section{ACKNOWLEDGMENT}

This work was performed at Microsoft Research Asia.

\section{REFERENCES}

[1] "Requirements for a 3G Network Based on Internet Protocol ("All-IP") With Support for TIA/EIA-41 Interoperability," 3GPP2 specification, 2000.

[2] V. Varsa and M. Karczewicz, "Common Conditions for 3GPP/3GPP2 Conversational and Streaming Over RTP/IP," ITU-T Study Group 16, VCEG-M55, 2001.

[3] T. Moriya, N. Iwakami, J. Akio, and T. Mori, "A design of lossy and lossless scalable audio coding," in Proc. ICASSP'00, vol. 2, 2000, pp. 889-892.

[4] P. Kudumakis and M. Sandler, "Wavelet packet based scalable audio coding," in Proc. ISCAS'96, vol. 2, 1996, pp. 41-44.

[5] T. S. Rappaport, Wireless Communications Principles and Practice. Englewood Cliffs, NJ: Prentice-Hall, 1996.

[6] I. Moccagatta, S. Soudagar, J. Liang, and H. Chen, "Error-resilient coding in JPEG-2000 and MPEG-4," IEEE J. Select. Areas Commun., vol. 18, pp. 899-914, June 2000.

[7] C. Yung, H. Fu, C. Tsui, R. S. Cheng, and D. George, "Unequal error protection for wireless transmission of MPEG audio," in Proc. 1999 IEEE Int. Symp. Circuits and Systems 1999 (ISCAS '99), vol. 6, 1999.

[8] J. Hagenauer and T. Stockhammer, "Channel coding and transmission aspects for wireless multimedia," IEEE J. Select. Areas Commun., vol. 10, pp. 1764-1777, Oct. 1999.

[9] J. Bolot and A. Vega-Garcia, "The case for FEC-based error control for packet audio in the internet," in Proc. ACM Multimedia Systems' 97 , **AUTHOR: PLS. PROVIDE PAGES/LOCATION**.

[10] J. Bolot, S. Fosse-Parisis, and D. Towsley, "Adaptive FEC-based error control for interactive audio in the internet," in Proc. IEEE Infocom' 99, New York, Mar. 1999.

[11] D. G. Sachs, R. Anand, and K. Ramchandran, "Wireless image transmission using multiple-description based concatenated codes," in Proc. DCC 2000, Snowbird, UT.

[12] P. Cosman, J. Rogers, P. G. Sherwood, and K. Zeger, "Image transmission over channels with bit errors and packet erasures," in Proc. 32nd Asilomar Conf. Signals, Systems, and Computers, Monterey, CA, Nov. 1998.

[13] J. Zhou, Q. Zhang, Z. Xiong, and W. Zhu, "Error resilient scalable audio coding (ERSAC) for mobile applications," in IEEE Workshop on Multimedia Signal Processing 2001, Cannes, France, Oct. 2001.

[14] "Report on the MPEG-4 Audio Version 2 Verification Test," MPEG w3075, 1999.

[15] T. Painter and A. Spanias, "Perceptual coding of digital audio," Proc. IEEE, vol. 88, pp. 451-513, Apr. 2000.

[16] M. Zorzi, R. R. Rao, and L. B. Milstein, "On the accuracy of a fist-order Markov model for data block transmission on the fading channels," in Proc. IEEE ICUPC'95, Tokyo, Japan, Nov. 1995.

[17] Q. Zhang, W. Zhu, and Y.-Q. Zhang, "Channel-adaptive resource allocation for scalable video transmission over $3 \mathrm{G}$ wireless network," IEEE Trans. Circuits Syst. Video Technol., vol. 14, pp. 1049-1063, Aug. 2004.

[18] M. Zorzi, R. R. Rao, and L. B. Milstein, "Error statistics in data transmission over fading channels," IEEE Trans. Commun., vol. 46, pp. 1468-1477, Nov. 1998.

[19] T. Takishima, M. Wada, and H. Murakami, "Reversible variable length codes," IEEE Trans. Commun., vol. 43, no. 2/3/4, pp. 158-162, 1995.

[20] G. Wen and J. Villasenor, "A class of reversible variable length codes for robust image and video coding," in Proc. ICIP '97, vol. 2, Santa Barbara, CA, Oct. 1997, pp. 65-68.

[21] R. Talluri, "Error resiliency in ISO's MPEG-4 video coding standard," IEEE Commun. Mag., vol. 36, pp. 112-119, June 1998. 
[22] J. Teuhola, "A compression method for clustered bit-vectors," Inform. Processing Lett., vol. 7, pp. 308-311, Oct. 1978.

[23] S. Lin and D. J. Costello Jr, Error Control Coding: Fundamentals and Applications. Englewood Cliffs, NJ: Prentice-Hall, 1983.

[24] S. I. Arazaki, M. Saigusa, S. Hashiguchi, M. Ohki, M. Uchiyama, and F. Itoh, "Image data compression by DCT with adaptive run-length coding," IEEE Trans. Consumer Electron., vol. 37, Nov. 1991.

[25] G. Cheung and A. Zakhor, "Optimal bit allocation for joint source/channel coding of scalable video," IEEE Trans. Image Processing, vol. 9, pp. 340-357, Mar. 2000.

[26] K. Ramchandran, A. Ortega, and M. Vetterli, "Bit allocation for dependant quantization with applications to multiresolution and MPEG video coders," IEEE Trans. Image Processing, vol. 37, pp. 533-545, Aug. 1994.

[27] R. Beaton et al., "Objective perceptual measurement of audio quality," in Digital Audio Bit-Rate Reduction, N. Gilchrist and C. Grewin, Eds: Audio Engineering Society, 1996, pp. 126-152.

[28] "Selection Procedures for the Choice of Radio Transmission Technologies of the UMTS," UMTS 30.03, 1998.

[29] J. Woller. (1996) The Basics of Monte Carlo Simulations. [Online]. Available: http://wwitch.unl.edu/zeng/joy/mclab/mcintro.html

[30] H. Cai and G. Mirchandani, "Wavelet transform and bit-plane encoding," in Proc. IEEE Int. Conf. Image Processing, Oct. 1995.

[31] "QoS Concepts and Architecture," 3GPP TS 23.107, 2002.

[32] R. Fletcher, Practical Methods of Optimization, 2nd ed. New York: Wiley, 1987.

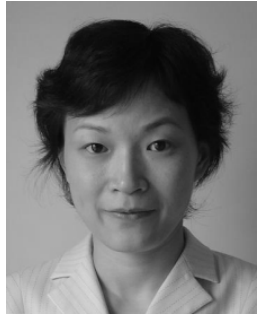

Qian Zhang (M'00) received the B.S., M.S., and Ph.D. degrees from Wuhan University, China, in 1994, 1996, and 1999, respectively, all in computer science.

She joined Microsoft Research, Asia, Beijing, China, in July 1999. Now, she is the research manager of the Wireless and Networking Group. She has published about 80 refereed papers in international leading journals and key conferences in the areas of wireless/Internet multimedia networking, wireless communications and networking, and overlay networking. She is the inventor of about 20 pending patents. Her current research interest includes seamless roaming across different wireless networks, multimedia delivery over wireless, Internet, next-generation wireless networks, P2P network/ad hoc network. She also participated many activities in the IETF ROHC (Robust Header Compression) WG group for TCP/IP header compression.

Dr. Zhang is a member of the Visual Signal Processing and Communication Technical Committee and the Multimedia System and Application Technical Committee of the IEEE Circuits and Systems Society. She is also a member and chair of QoSIG of the Multimedia Communication Technical Committee of the IEEE Communications Society. She is now serving as Associate Editor of IEEE TRANSACTIONS ON VeHICULAR TECHNOLOGY. She is also serving as Guest Editor for special issue on wireless video in IEEE Wireless Communication Magazine. Meanwhile, she is organizing two special sessions in IEEE ICC 2005 and IEEE ISCAS 2005, respectively. She has recently received the TR 100 (MIT Technology Review) world's top young innovator award.

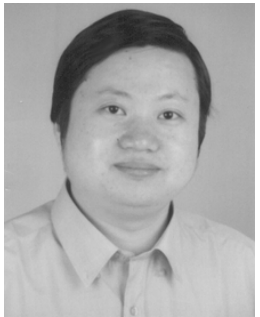

Guijin Wang received the B.S. degree in electronic engineering in 1998 from Tsinghua University, Beijing, China, where he is currently working toward $\mathrm{Ph}$.D. degree in electronic engineering.

From 1999 to 2002, he was a Research Member of Image Communication and Processing Lab, Tsinghua University. During this period, he was a Visiting Student of the Wireless and Networking Group, Microsoft Research Asia, Beijing, China. His current research interests include multimedia delivery over wireless, the Internet, video (H264) coding, next-generation wireless networks, and video processing.
Zixiang Xiong (M'00-SM'04) received the Ph.D. degree in electrical engineering from the University of Illinois at Urbana-Champaign in 1996.

From 1997 to 1999, he was with the University of Hawaii. Since 1999, he has been with the Department of Electrical Engineering, Texas A\&M University, College Station, where he is an Associate Professor. He spent the summers of 1998 and 1999 at Microsoft Research, Redmond, WA, and the summers of 2000 and 2001 at Microsoft Research Asia, Beijing, China. His current research interests are joint source-channel coding, distributed source coding, genomic signal processing, and network traffic smoothing and modeling.

Dr. Xiong is currently an Associate Editor for the IEEE TRANSACTIONS ON CiRCUITS AND SYSTEMS FOR VIDEO TECHNOLOGY, the IEEE TRANSACTIONS ON Signal Processing, and the IEEE TRANSACTIONS ON IMAGe Processing. He received a National Science Foundation Career Award in 1999, an ARO Young Investigator Award in 2000, and an ONR Young Investigator Award in 2001. $\mathrm{He}$ also received an Eugene Webb Faculty Fellow Award in 2001 and a Select Young Faculty Award in 2002, both from Texas A\&M University.

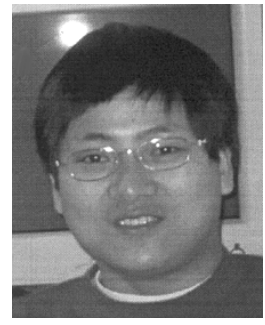

Jianping Zhou received the B.S. degree in applied mathematics from Xi' an Jiaotong University, Xi'an, China, in 1997 and the M.E. degree in signal processing from Peking University, Peking, China in 2000. He is currently pursuing the Ph.D. degree in electrical engineering at the University of Illinois at Urbana-Champaign. His research interests include digital signal processing, multimedia coding and streaming, multirate systems, and wavelet transforms.

China, from 2000 to 2002.

He was with Microsoft Research Asia, Beijing,

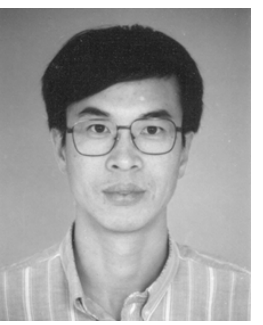

Wenwu Zhu (S'96-M'96-SM'01) received the B.E. and M.E. degrees from the National University of Science and Technology, China, in 1985 and 1988 , respectively, the M.S. degree from Illinois Institute of Technology, Chicago, and the Ph.D. degree from Polytechnic University, Brooklyn, NY, in 1993 and 1996, respectively, all in electrical engineering.

From August 1988 to December 1990, he was with the Graduate School, University of Science and Technology of China (USTC), and the Institute of Electronics, Chinese Academy of Sciences, Beijing, China. He joined Microsoft Research Asia, Beijing, in 1999 as a Researcher in the Internet Media Group. He is currently a Research Manager of the Wireless and Networking Group. Prior to his current post, he was with Bell Labs, Lucent Technologies, Murray Hill, NJ, as a Member of Technical Staff during 1996-1999. While he was with Bell Labs, he performed research and development in the areas of Internet video, video conferencing, and video streaming over IP networks. He has published over 100 refereed papers in international leading journals and key conferences in the areas of wireless/Internet video transport, wireless/Internet multimedia communications and networking, and multimedia signal processing, and has contributed to the IETF ROHC WG draft on robust TCP/IP header compression over wireless links. He is the inventor of more than a dozen pending patents. His current research interest is in the area of wireless/Internet multimedia delivery and delivery networks.

Dr. Zhu has served as Guest Editor for Special Issues on Streaming Video and Wireless Video in IEEE TRANSACTIONS ON CIRCUITS AND SYSTEMS FOR VIDEO TECHNOLOGY (TCSVT). He received the Best Paper Award in IEEE TCSVT in 2001. He is a member of Eta Kappa Nu, the Visual Signal Processing and Communication Technical Committee, and the Multimedia System and Application Technical Committee of the IEEE Circuits and Systems Society. He is also a member of the Multimedia Communication Technical Committee of the IEEE Communications Society. 